\title{
Stable viscous shocks in elliptic conservation laws
}

\author{
Mariana Haragus \\ Université de Franche-Comté \\ Département de Mathématiques \\ 16 route de Gray \\ 25030 Besançon cedex, France \\ Arnd Scheel \\ University of Minnesota \\ School of Mathematics \\ 206 Church St. S.E. \\ Minneapolis, MN 55455, USA
}

\begin{abstract}
We study quadratic systems of viscous conservation laws which arise as long-wavelength modulation equations near planar, modulated traveling waves. In [3], we showed that the conservation law is either elliptic or hyperbolic in a full neighborhood of the origin. Moreover, in parameter space the ill-posed, elliptic inviscid limit coincides with the robust occurrence of localized degenerate viscous shocks that correspond to localized spikes in the profile of the traveling wave. We refer to these localized degenerate shock waves as holes. In this paper, we study a special case, where the effective viscosity in the conservation law is a scalar. Although the ellipticity of the underlying inviscid conservation law creates an instability of the linearized transport equation at every single point of the degenerate shock wave, which moreover is absolute in a region near the shock location, holes and accompanying overcompressive shocks turn out to be asymptotically stable. We conclude with an example of a reaction-diffusion system with a planar modulated wave where our results predict the existence of families of stable holes in the planar front.
\end{abstract}

Acknowledgments This work was partially supported by the Ministère de la Recherche through grant ACI JC 1039 (M. H.) and by the National Science Foundation through grant NSF DMS-0504271 (A. S.).

Running head: Viscous shocks in elliptic conservation laws

Keywords: corners in interfaces, viscous conservation laws, shocks, stability 


\section{Introduction}

Traveling-wave solutions are particular solutions to translation-invariant systems, which can often be studied analytically, or at modest numerical expense, but yet provide insight in the dynamics of spatially extended systems. Traveling waves also serve as building blocks for more complicated spatio-temporal patterns. In recent work $[3,4,5]$, we studied traveling waves in planar reaction diffusion systems: assuming existence and certain stability properties of a planar traveling wave, that is, a wave which does not depend on one of the coordinates, $U\left(x_{1}, x_{2}, t\right)=U_{*}\left(x_{1}-c t\right)$, say, we found a plethora of traveling waves, which resemble the primary front along each line $x_{2}=$ const, but shifted by a phase $U \sim U_{*}\left(x_{1}-c t-\xi\left(x_{2}\right)\right)$. Of particular interest are corners in interfaces, where $\xi^{\prime}\left(x_{2}\right)=\eta\left(x_{2}\right) \rightarrow \eta_{ \pm}$as $x_{2} \rightarrow \pm \infty$. We found exterior corners, $\eta_{-}<\eta_{+}$, interior corners, $\eta_{-}>\eta_{+}$, and steps, $\eta_{-}=\eta_{+} \neq 0$. The situation is much richer when the traveling wave propagates in an oscillatory fashion, $U\left(x_{1}, x_{2}, t\right)=U_{\mathrm{mtw}}\left(x_{1}-c t, \omega t\right)=U_{\mathrm{mtw}}\left(x_{1}-c t, \omega t+2 \pi\right)$. Then in addition to corners, we found asymptotically flat shapes $\xi\left(x_{2}\right)$ with $\xi^{\prime}\left(x_{2}\right) \sim 1 /\left|x_{2}\right|$ as $x_{2} \rightarrow \pm \infty$. We refer to these interfaces as holes since they typically cause a localized depression in the (almost planar) level set of the traveling wave. Figure 1.1 illustrates the different kinds of corners in a numerical simulation. Plotted is the value of one of the chemical species; the planar wave trains are generated at the boundary and travel upwards. In the left-hand and in the middle picture, the planar interface of the wave trains is perturbed by a vertical line inhomogeneity along which exterior corners and holes form, respectively. Also visible in the left-hand picture are interior corners to the left- and right-hand sides of the exterior corners, forming the corner between the horizontal wave fronts in the far field and the outward propagating wave fronts emanating from the exterior corner. In the right-hand picture, the line inhomogeneity travels with a constant speed to the right in a frame moving upwards with the interface and the interface forms a step across the inhomogeneity. See [5, Section 7] for more details.
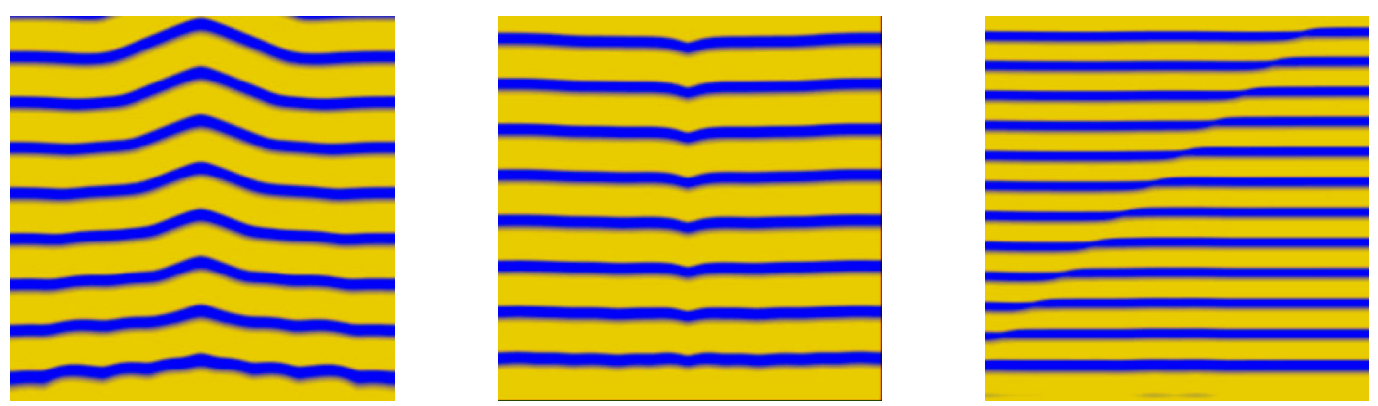

Figure 1.1: Snapshots of numerical simulations of traveling waves in an oscillatory medium with a line inhomogeneity; see the text for further details.

A related view on the dynamics of traveling waves focusses on large-scale modulations of the position $\xi\left(x_{2}\right)$. An Ansatz $U=U_{*}\left(x_{1}-c t+\xi\left(\varepsilon x_{2}, \varepsilon^{2} t\right)\right)+\mathrm{O}\left(\varepsilon^{2}\right)$ yields an Eikonal equation

$$
\xi_{T}=d \xi_{X X}-g\left(\xi_{X}\right), \quad T=\varepsilon^{2} t, X=\varepsilon x_{2},
$$

as a compatibility condition in a formal expansion of the system in terms of $\varepsilon$. Here, the 
constant $d$ is an effective viscosity, and the flux $g$ is quadratic, determined by geometry. For modulated waves, the Ansatz can be adapted to

$$
U=U_{\mathrm{mtw}}\left(x_{1}-c t+\xi\left(\varepsilon x_{2}, \varepsilon^{2} t\right), \omega t+\theta\left(\varepsilon x_{2}, \varepsilon^{2} t\right)\right)+\mathrm{O}\left(\varepsilon^{2}\right)
$$

and gives a system of equations

$$
\left(\begin{array}{c}
\xi_{T} \\
\theta_{T}
\end{array}\right)=d\left(\begin{array}{c}
\xi_{X X} \\
\theta_{X X}
\end{array}\right)-\left(\begin{array}{c}
g_{1}\left(\xi_{X}, \theta_{X}\right) \\
g_{2}\left(\xi_{X}, \theta_{X}\right)
\end{array}\right) .
$$

Here, $d$ is a $2 \times 2$-matrix, the effective viscosity, and the fluxes $g_{j}$ are quadratic. With variables $\eta=\xi_{X}$ and $\rho=\theta_{X}$, this system becomes a quadratic viscous conservation law. Corners as described above correspond to viscous shocks, and slopes $\xi_{X}=\eta$ and $\theta_{X}=\rho$, determine characteristic speeds at infinity. In this regard, holes are of particular interest as they correspond to degenerate but robust viscous shocks. Since slopes $\xi_{X}$ and frequency $\theta_{X}$ vanish asymptotically, both asymptotic characteristic speeds are glancing modes, moving parallel to the shock location. These shocks occur in two-parameter families, parameterized by position and amplitude, for an open set of fluxes $g_{j}$.

It turns out that holes (or degenerate shocks) exist precisely when the system of conservation laws for $\eta$ and $\rho$ is elliptic in the inviscid limit $d=0$, which amounts to certain conditions on the quadratic fluxes. One might therefore conjecture that these robust degenerate shocks are unstable since the inviscid limit is highly unstable, ill-posed as an initial-value problem. In this article we investigate shocks in the viscous conservation laws with elliptic inviscid limit, under the assumption that the effective viscosity is a scalar, $d=d_{0}$ id. Our main result shows that degenerate shocks (and hence holes) are asymptotically stable.

Traveling waves arise in a number of physical systems, often as fronts $U_{*}\left(x_{1}-c t\right) \rightarrow u_{ \pm}$ for $x_{1} \rightarrow \pm \infty$ or pulses $U_{*}\left(x_{1}-c t\right) \rightarrow 0$ for $x \rightarrow \pm \infty$, or as spatially periodic wave trains $U_{*}\left(x_{1}-c t\right)=U_{*}\left(x_{1}-c t+L\right)$. Modulated waves arise via Hopf bifurcation from fronts in combustion problems, or from wave trains in convection experiments [9]. As an application of our results, we construct an explicit example of a front undergoing a Hopf bifurcation, such that the bifurcating modulated front gives rise to a system of conservation laws with scalar effective viscosity, and an ill-posed elliptic inviscid limit.

The remainder of this article is organized as follows. We review the derivation of the system of conservation laws and gather properties of the fluxes in Section 2. We then briefly review stability properties of the constant solutions to the viscous elliptic conservation law in Section 3 , and the existence of holes in Section 4. In particular, we show that the linearization at every point of a hole is unstable, and this instability is absolute for the points near the top of the hole. We then show that holes can be stable: in the simplest case of scalar effective viscosity, $d=d_{0}$ id, holes are spectrally stable under $L^{2}$-perturbations (Section 5 ) and nonlinearly stable for perturbations with zero mass (Section 6). We conclude with a brief summary of other possible shocks (Section 7), an explicit construction of an example (Section 8), and a short discussion (Section 9). 


\section{From traveling waves to conservation laws}

Consider a modulated planar traveling-wave solution $U_{\mathrm{mtw}}\left(x_{1}-c_{*} t, \omega_{*} t\right)=U_{\mathrm{mtw}}\left(x_{1}-c_{*} t, \omega_{*} t+\right.$ $2 \pi)$ in an isotropic reaction-diffusion system

$$
U_{t}=D \triangle U+f(U), \quad U \in \mathbb{R}^{N}, x=\left(x_{1}, x_{2}\right) \in \mathbb{R}^{2},
$$

with smooth kinetics $f \in C^{\infty}$ and positive diffusion matrix $D=D^{*}>0$. We can think of a modulated wave train $U_{\mathrm{mtw}}(\zeta, \tau)=U_{\mathrm{mtw}}(\zeta+L, \tau)$, or a pulsating front $U_{\mathrm{mtw}}(\zeta, \tau) \rightarrow U_{ \pm}$for $\zeta \rightarrow \pm \infty$. In the following, we concentrate on the case of a pulsating front, the case of a wave train being similar.

Substituting the Ansatz

$$
U\left(x_{1}, x_{2}, t\right)=U_{\mathrm{mtw}}\left(x_{1}-c t+\xi\left(\varepsilon x_{2}, \varepsilon^{2} t\right), \omega t+\theta\left(\varepsilon x_{2}, \varepsilon^{2} t\right)\right)+\varepsilon^{2} V\left(x_{1}-c t, \omega t, \varepsilon x_{2}, \varepsilon^{2} t\right),
$$

into the reaction-diffusion system, leads to a viscous conservation law

$$
u_{T}=d u_{X X}+(A(u, u))_{X}, \quad T=\varepsilon^{2} t, X=\varepsilon x_{2},
$$

where $u=(\eta, \rho)=\left(\xi_{X}, \theta_{X}\right), d$ is a $2 \times 2$-matrix, and $A$ a bilinear form. While we do not attempt a rigorous derivation and justification of this modulation equation, we refer to $[3$, Appendix 2] for a formal derivation in the present context and to [2] for a justification on large time scales for a somewhat simpler, one-dimensional problem with a reduced scalar conservation law. However, the derivation of the underlying steady system

$$
d u_{X}+A(u, u)=C,
$$

is completely justified by a center manifold reduction [3, Section 5]. In the following, we will interpret coefficients in this system of conservation laws and derive properties of the flux $A$.

First, the matrix $d$ relates to stability properties of the modulated wave. In the space of $x_{2}$-independent functions, both time and space derivatives of the modulated wave contribute to the kernel of the linearization. In two space dimensions, Fourier transform in $x_{2}$ allows to continue this neutral eigenspace with a Lyapunov-Schmidt-reduced linearization $-d k^{2}+\mathrm{O}\left(k^{4}\right)$, where $d$ is a two-dimensional effective diffusion matrix and $k$ the Fourier variable; see [3, Section 5] for more precise assumptions. Of course, the reflection symmetry of the underlying equation together with the isotropy of the modulated wave enforce the reduced linearization to be an even function of $k$. We are interested in linearly stable waves, where Respec $d>0$.

Next, the conservation form of the system generates a two-parameter family of equilibria with $A(u, u)=\left(c^{\prime}, \omega^{\prime}\right)^{T}$. The constants $c^{\prime}$ and $\omega^{\prime}$ are corrections to speed and frequency of the modulated waves,

$$
c^{\prime}=c_{*}-c, \quad \omega^{\prime}=\omega-\omega_{*},
$$

and act as free parameters [3, Section 5]. In the reaction-diffusion system, this family is generated as follows. First, since the medium is isotropic, any rotated wave $U_{\mathrm{mtw}}\left(x \cdot n-c_{*} t, \omega_{*} t\right)$ with $n=(\cos \varphi, \sin \varphi)$ is a solution. Second, the solution is accompanied by solutions with 
periodic modulations in the transverse direction, $U \sim U_{\mathrm{mtw}}\left(x_{1}-c t, \omega t-k x_{2}\right)$, with $\omega=\omega(k)$ and $c=c(k)$ for $k$ small. Indeed, the periodic modulations satisfy

$$
\omega U_{\tau}-D k^{2} U_{\tau \tau}=D U_{\zeta \zeta}+f(U)+c U_{\zeta}, \quad \tau=\omega t-k x_{2}, \zeta=x_{1}-c t,
$$

which can be rewritten as an $\mathrm{O}\left(k^{2}\right)$-perturbation of the equation for the planar modulated wave

$$
\begin{gathered}
\omega U_{\tau}-\left(D U_{\zeta \zeta}+f(U)+c U_{\zeta}\right)-\left(\left(\mathrm{id}-D \frac{k^{2}}{\omega} \partial_{\tau}\right)^{-1}-\mathrm{id}\right)\left(D U_{\zeta \zeta}+f(U)+c U_{\zeta}\right) \\
=\omega U_{\tau}-\left(D U_{\zeta \zeta}+f(U)+c U_{\zeta}\right)+\mathrm{O}\left(k^{2}\right),
\end{gathered}
$$

so that we can solve near the planar wave for $\omega$ and $c$ as functions of $k^{2}$.

We showed in [3] that the flux possesses the particular form

$$
A(u, u)=c_{*}\left(\begin{array}{c}
-\frac{1}{2} \eta^{2}+\alpha_{1} \rho^{2} \\
-\eta \rho+\alpha_{2} \rho^{2}
\end{array}\right), \quad \alpha_{1}, \alpha_{2} \in \mathbb{R} .
$$

We argue here that this particular form can be deduced from the action of the Euclidean group, only. The general form of a quadratic flux is

$$
A(u, u)=\left(\begin{array}{c}
a_{1} \eta^{2}+a_{2} \eta \rho+a_{3} \rho^{2} \\
b_{1} \eta^{2}+b_{2} \eta \rho+b_{3} \rho^{2}
\end{array}\right) .
$$

Our claim is that $a_{1}=b_{2} / 2=-c_{*} / 2$ and $a_{2}=b_{1}=0$, solely because of the rotational symmetry. Indeed, we may consider the rotated profile $\eta=\eta_{*}, \rho=0$. In rotated coordinates, the linearization would be symmetric, with vanishing linear flux. When we transform back to the original coordinates, we generate a flux in the direction $x_{2}$ along the interface, given by a scalar, proportional to the angle. Indeed, transport in the rotated coordinates is by reflection symmetry only in the normal direction to the interface, with normal speed of propagation $c_{*}$ (or $c_{\mathrm{g}}$ in case of a wave train). The direction normal to the interface in the rotated coordinates decomposes into a changed normal speed of propagation and transport along the interface proportional to the angle $\eta_{*}$. Therefore, the linearization of $A(u, u)$ in a point $u_{*}=\left(\eta_{*}, 0\right)$ is given by

$$
\frac{\mathrm{d}}{\mathrm{d} u} A\left(u_{*}, u_{*}\right) \widetilde{u}=2 A\left(\widetilde{u}, u_{*}\right)=\left(\begin{array}{l}
-c_{*} \eta_{*} \widetilde{\eta} \\
-c_{*} \eta_{*} \widetilde{\rho}
\end{array}\right) .
$$

Comparing with the general form of $A$ now proves the claim.

Finally, notice that after a scaling in $\eta$ and $\rho$, we have

$$
A(u, u)=\left(\begin{array}{c}
-\frac{1}{2} \eta^{2}+\alpha_{1} \rho^{2} \\
-\eta \rho+\alpha_{2} \rho^{2}
\end{array}\right), \quad \alpha_{1}, \alpha_{2} \in \mathbb{R} .
$$

In addition, the shear transformation $\tilde{\eta}=\eta-\alpha_{2} \rho$ succeeded by a scaling $\tilde{\rho}=\sqrt{\left|2 \alpha_{1}-\alpha_{2}^{2}\right|} \rho$ gives the simplified flux

$$
A(u, u)=\left(\begin{array}{c}
-\frac{1}{2} \eta^{2} \pm \frac{1}{2} \rho^{2} \\
-\eta \rho
\end{array}\right)
$$


where the sign \pm corresponds to the sign of $2 \alpha_{1}-\alpha_{2}^{2}$. Also notice that the special case of scalar effective viscosity, $d=d_{0}$ id, reduces to the case of trivial diffusion $d=$ id after a scaling in $T$ and $X$.

\section{Elliptic conservation laws and absolute instability of fre- quency modulations}

Stability and well-posedness in conservation laws crucially depends on the characteristic speeds. A short computation of the eigenvalues of $A(u, \cdot)$ shows that in the zero-viscosity limit the system (2.1) with particular quadratic form $A$ as in (2.3) is

- hyperbolic, real characteristic speeds, if $2 \alpha_{1}<\alpha_{2}^{2}$;

- elliptic, complex characteristic speeds, if $2 \alpha_{1}>\alpha_{2}^{2}$.

In the hyperbolic case, $2 \alpha_{1}<\alpha_{2}^{2}$, we may diagonalize the simplified flux (2.4) with $v_{1}=\eta+\rho$ and $v_{2}=\eta-\rho$, and then find two real viscous Burgers' equations,

$$
v_{j t}=\sum_{k=1,2} \tilde{d}_{j k} v_{k x x}-v_{j} v_{j x}, \quad j=1,2
$$

(here, and in the remainder of the paper, we use the notation $x$ instead of $X$ ). On the other hand, when $2 \alpha_{1}>\alpha_{2}^{2}$, we set $Z=\eta+\mathrm{i} \rho$, and obtain

$$
Z_{t}=\tilde{d}_{1} Z_{x x}+\tilde{d}_{2} \bar{Z}_{x x}-Z Z_{x}
$$

In the special case $d=\mathrm{id},(3.1)$ and (3.2) simplify to two uncoupled Burgers' equations

$$
v_{j t}=v_{j x x}-v_{j} v_{j x}, \quad j=1,2,
$$

and the complex continuation of Burgers' equation

$$
Z_{t}=Z_{x x}-Z Z_{x}
$$

respectively.

While (3.1) and (3.3) give hyperbolic conservation laws in the inviscid limit, (3.2) and (3.4) possess complex characteristics with speed $Z$ complex for $Z \notin \mathbb{R}$, that is, for nonzero frequency modulations $\rho \neq 0$. In particular, the linearization at any state $u \equiv$ const is stable in the hyperbolic case and unstable in the elliptic case provided $\rho \neq 0$. In addition, the instability of the linearized equation

$$
Z_{t}=Z_{x x}-Z_{*} Z_{x}
$$

is in fact absolute $[1,10]$ when $\operatorname{Re} Z_{*}^{2}<0$, that is, when $\left|\operatorname{Re} Z_{*}\right|<\left|\operatorname{Im} Z_{*}\right|$. To see this, note that the dispersion relation $\lambda=\nu^{2}-Z_{*} \nu$ of the linearization possesses a double root at $\lambda=-Z_{*}^{2} / 4$ : after transforming $Z=Y \mathrm{e}^{Z_{*} x / 2}$, we find the heat equation with linear growth

$$
Y_{t}=Y_{x x}-\frac{Z_{*}^{2}}{4} Y
$$


It is not difficult to see that even for general diffusion matrices, there always exists a conical region of absolute instability.

Quadratic hyperbolic conservation laws have been studied in great generality [12]. It is also known that non-Lax shocks can occur and be stable [7]. For elliptic conservation laws, absolute instability has been shown in a general setup, including constants in the above complex continuation of the Burgers' equation [6]. We focus here on stability of shocks in the elliptic conservation law (3.4). Notice that this complex continuation of Burgers' equation is different from the complex Burgers' equation in [7], which is hyperbolic in the inviscid limit.

\section{Localized viscous shock profiles}

We showed in [3, Section 5] that holes exist as two-parameter families for the full reactiondiffusion system whenever the inviscid conservation law falls into the elliptic regime. In the viscous conservation law (2.1), hole solutions correspond to localized waves which satisfy the quadratic ODE

$$
u_{x}=-d^{-1} A(u, u) .
$$

Quadratic ODEs in the plane have been studied in great generality [8]. For our system the results in [3, Section 5] show that localized waves exist precisely in the elliptic regime $\alpha_{2}^{2}<2 \alpha_{1}$. Such solutions always approach zero along ray solutions,

$$
u(x)=\frac{u_{*}}{x},
$$

in which $u_{*} \in \mathbb{R}^{2}$ is a fixed point of $d^{-1} A$,

$$
u_{*}=d^{-1} A\left(u_{*}, u_{*}\right) \text {. }
$$

In particular, they decay algebraically as $|x| \rightarrow \infty$. Figure 4.1 shows four typical phase portraits for the quadratic ODE in the elliptic case, $\alpha_{2}^{2}<2 \alpha_{1}$, and hyperbolic case, $\alpha_{2}^{2}>2 \alpha_{1}$. In both cases, the ODE has either one or three ray solutions, since $A$ is a quadratic form.

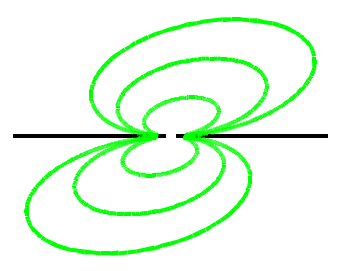

(a)

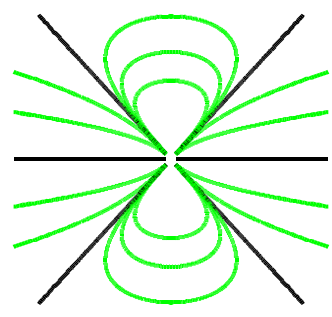

(b)

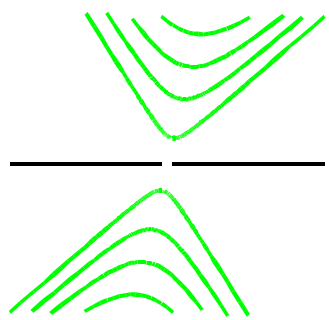

(c)

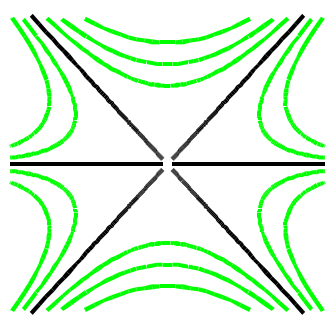

(d)

Figure 4.1: Typical phase portraits of (4.1) in the elliptic case (a-b), and hyperbolic case (c-d). The black lines represent the ray solutions. 
From now on we restrict to the case of a scalar effective viscosity, $d=d_{0}$ id, when the system is equivalent to the Burgers equation (3.4). Then holes satisfy the complex-valued ODE

$$
Z_{x}=\frac{1}{2} Z^{2}
$$

and are given explicitly by the two-parameter family

$$
Z_{*}(x)=-\frac{2}{x+\mathrm{i} a+b}, \quad a \in \mathbb{R}^{*}, b \in \mathbb{R} .
$$

The limit $a=0$ corresponds to the unique (up to translations in $x$ ) ray solution which is unbounded and has a pole at the origin. According to Section 3 values close to the top of the wave lie in the region of absolute instability, while asymptotic values lie in the region of absolute stability; see Figure 4.2. Nevertheless, we prove in the next two sections that these waves are stable.

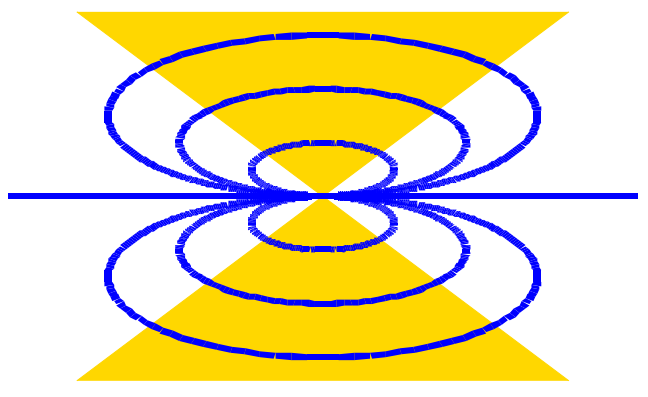

Figure 4.2: Phase portrait of (4.2) and the region of absolute instability (shaded).

\section{$5 \quad$ Spectral stability of holes}

The linearization of (3.4) at a hole $Z_{*}$ gives the linear operator

$$
\mathcal{L} Z=Z_{x x}-\left(Z_{*}(x) Z\right)_{x}, \quad Z_{*}(x)=-\frac{2}{x+\mathrm{i} a+b}, \quad a \neq 0,
$$

that we consider here in $L^{2}(\mathbb{R})$. Since $Z_{*}(x)=\mathrm{O}(1 /|x|)$ as $|x| \rightarrow \infty$, this operator is a relatively compact perturbation of the Laplacian on $L^{2}(\mathbb{R})$, so that its essential spectrum is given by $\operatorname{spec}_{\text {ess }} \mathcal{L}=(-\infty, 0]$. In order to determine the point spectrum, we have to study the eigenvalue problem,

$$
\lambda Z=Z_{x x}+\frac{2}{x+\mathrm{i} a+b} Z_{x}-\frac{2}{(x+\mathrm{i} a+b)^{2}} Z,
$$

which is a Bessel-type equation. Upon setting $\lambda=-\gamma^{2}, z=\gamma(x+\mathrm{i} a+b)$, and

$$
Z(x)=(x+\mathrm{i} a+b)^{-\frac{1}{2}} W(z),
$$


we find the standard Bessel equation

$$
W_{z z}+\frac{1}{z} W_{z}+\left(1-\frac{9}{4} \frac{1}{z^{2}}\right) W=0 .
$$

Two linearly independent solutions of this equation are given by the Hankel functions $H_{\frac{3}{2}}^{(1)}$ and $H_{\frac{3}{2}}^{(2)}$, which are holomorphic in the complex plane cut along the negative real axis, and have the asymptotic behavior

$$
H_{\frac{3}{2}}^{(1)} \sim \frac{\sqrt{2}}{\sqrt{\pi z}} \mathrm{e}^{\mathrm{i}(z-\pi)}, \quad H_{\frac{3}{2}}^{(2)} \sim \frac{\sqrt{2}}{\sqrt{\pi z}} \mathrm{e}^{-\mathrm{i}(z-\pi)}
$$

as $|z| \rightarrow \infty,|\arg z|<\pi$.

For $\operatorname{Re} \lambda>0$, we choose $\gamma$ such that $a \operatorname{Im} \gamma<0$. Then the two Hankel functions above provide us with two linearly independent solutions to (5.1), which are analytic in $x$, since $\gamma(x+\mathrm{i} a+b) \notin \mathbb{R}_{-}$, for any real $x$. The asymptotic behavior of the Hankel functions then shows that these two solutions are unbounded, so that (5.1) has no bounded solutions for $\operatorname{Re} \lambda>0$. This proves the absence of point spectrum in the right-half complex plane, and we may conclude that holes are spectrally stable. More precisely, we have the following result.

Theorem 1 Consider a hole solution $Z_{*}$ to (3.4) with $a \neq 0$. Then the spectrum of the linearized operator

$$
\mathcal{L} Z=Z_{x x}-\left(Z_{*}(x) Z\right)_{x}
$$

in $L^{2}(\mathbb{R})$ entirely lies in the closed left-half complex plane. Consequently, the hole solution $Z_{*}$ is spectrally stable.

\section{Hopf-Cole and the dynamics of holes}

The dynamics of (3.4) can be studied using the complex extension of the Hopf-Cole transformation $Z=-2(\log \Psi)_{x}$, which gives the linear heat equation for $\Psi$,

$$
\Psi_{t}=\Psi_{x x}
$$

With this transformation the holes $Z_{*}$ yield solutions of the heat equation

$$
\Psi_{*}(x)=x+\mathrm{i} a+b .
$$

We can now use decay properties of the heat kernel to show nonlinear stability of holes for different classes of initial data. We illustrate this only in the very simple situation of the $\mathrm{O}(1 / \sqrt{t})$-decay in $L^{\infty}(\mathbb{R})$ of solutions to the heat equation with initial data in $L^{1}(\mathbb{R})$.

A convenient way of doing this transformation is by setting $Z=\Phi_{x}$ and then considering the complex continuation of the viscous eikonal equation

$$
\Phi_{t}=\Phi_{x x}-\frac{1}{2} \Phi_{x}^{2}
$$


to which the holes $Z_{*}$ give steady solutions

$$
\Phi_{*}(x)=-2 \log (x+\mathrm{i} a+b) .
$$

The following result for (6.2) implies the stability of the holes $Z_{*}$ for initial perturbations with zero mass and algebraic decay o $\left(1 /|x|^{3}\right)$ as $|x| \rightarrow \infty$.

Theorem 2 Consider a solution $\Phi_{*}$ to (6.2) with $a \neq 0$. Let $\Phi$ be the solution to (6.2) with initial data

$$
\left.\Phi\right|_{t=0}=\Phi_{*}+\phi_{0},
$$

such that

$$
\left\|(1+|x|) \phi_{0}(x)\right\|_{1}<\infty, \quad\left\|\phi_{0}\right\|_{\infty}<\infty .
$$

Then

$$
\left\|\Phi(t, \cdot)-\Phi_{*}\right\|_{\infty} \leq \frac{C}{\sqrt{t}}, \quad \forall t>1,
$$

for some positive constant $C$ (depending upon $\phi_{0}$ ). Here $\|\cdot\|_{1}$ and $\|\cdot\|_{\infty}$ represent the usual norms in $L^{1}(\mathbb{R})$ and $L^{\infty}(\mathbb{R})$, respectively.

Proof. We set $\Phi=\Phi_{*}+\phi$, and

$$
\Psi=\mathrm{e}^{-\frac{1}{2} \Phi}=\Psi_{*} \mathrm{e}^{-\frac{1}{2} \phi}=\Psi_{*}+\Psi_{*}\left(\mathrm{e}^{-\frac{1}{2} \phi}-1\right),
$$

where $\Psi_{*}(x)=x+\mathrm{i} a+b$, and $\Psi$ satisfies the heat equation (6.1). The perturbation

$$
\psi=\Psi_{*}\left(\mathrm{e}^{-\frac{1}{2} \phi}-1\right),
$$

also satisfies the heat equation, so that

$$
\psi(t, x)=\int_{\mathbb{R}} G(t, x-y) \Psi_{*}(y)\left(\mathrm{e}^{-\frac{1}{2} \phi_{0}(y)}-1\right) \mathrm{d} y,
$$

in which $G$ is the heat kernel. The properties of $\phi_{0}$ imply

$$
\Psi_{*}\left(\mathrm{e}^{-\frac{1}{2} \phi_{0}}-1\right) \in L^{1}(\mathbb{R}),
$$

so that from the decay properties of the heat kernel we conclude

$$
\|\psi(t, \cdot)\|_{\infty} \leq \frac{C_{1}}{\sqrt{t}}, \quad \forall t>0 .
$$

This inequality and the formula for $\psi$ imply the result in the theorem. 


\section{Other shocks}

We now briefly discuss the other types of shocks that exist in the case of trivial diffusion matrix. First notice that we can restrict to the case of vanishing shock speed due to the Galilei invariance of the quadratic flux. Next, in the hyperbolic case (3.3), all shocks are either constant in one component or classical Lax shocks. All shocks are stable. Combining two Lax shocks with equal speed gives stable overcompressive shocks.

In the elliptic case, shocks are solutions to the complex-valued ODE

$$
Z_{x}=\frac{1}{2} Z^{2}-\frac{1}{2} \alpha^{2}, \quad \alpha \in \mathbb{C}^{*}, \operatorname{Re} \alpha \geq 0 .
$$

For $\operatorname{Re} \alpha=0$ the dynamics of this ODE consists of periodic orbits surrounding the equilibria $Z_{ \pm}= \pm \alpha$, while for $\operatorname{Re} \alpha>0$ it consists of source-sink heteroclinics connecting the same equilibria $Z_{ \pm}= \pm \alpha$; see Figure 7.1. All these shocks are "overcompressive", with double
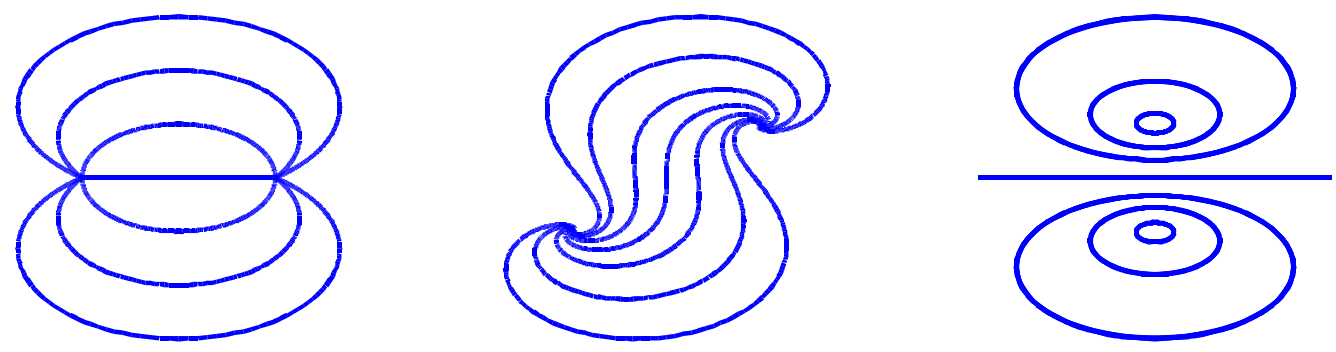

Figure 7.1: Phase portraits of (7.1) for $\alpha=1, \alpha=1+\mathrm{i}$, and $\alpha=\mathrm{i}$ (from left to right).

characteristic speed $2 \alpha$, and $-2 \alpha$, respectively. They are explicitly given by the family

$$
Z_{*}(x)=\alpha Z_{B}(\alpha(x+\beta)), \quad \beta \in \mathbb{C},
$$

where $Z_{B}$ denotes the classical real Burgers shock

$$
Z_{B}(x)=-\tanh \left(\frac{x}{2}\right) .
$$

The results in Section 3 show that equilibria $Z_{ \pm}= \pm \alpha$ with $\alpha \in \mathbb{R}$ are stable, and equilibria with $\alpha \notin \mathbb{R}$ are unstable with unstable essential spectrum. In addition, for $|\operatorname{Im} \alpha|>|\operatorname{Re} \alpha|$, the absolute spectrum destabilizes so that they are absolutely unstable.

For shocks, the linearized equation

$$
\lambda Z=Z_{x x}-\left(Z_{*} Z\right)_{x}
$$

transforms into

$$
\frac{\lambda}{\alpha^{2}} Z=Z_{y y}-\left(Z_{B} Z\right)_{y}, \quad y=\alpha(x+\beta) .
$$

Then for $\alpha \in \mathbb{R}$ we can use the stability of the Burgers shock to conclude that shocks with $\alpha \in \mathbb{R}$ are stable. Indeed, we have stability of the essential spectrum so that it is sufficient 
to exclude unstable point eigenvalues, which give necessarily exponentially localized solutions to the linearized equation (7.2). However, solutions to (7.2) are the analytic continuation of solutions to (7.3) in the complex time $y$, and exponential decay properties are preserved in the analytic continuation, so that eigenvalues $\lambda$ to (7.2) correspond to eigenvalues $\lambda / \alpha^{2}$ to (7.3).

Shocks with $\alpha \notin \mathbb{R}$ are unstable with unstable essential spectrum, due to the essential instability of the asymptotic states, and this instability is absolute for $|\operatorname{Im} \alpha|>|\operatorname{Re} \alpha|$ for the same reason. In the regime of essential but not absolute instability, $|\operatorname{Im} \alpha|<|\operatorname{Re} \alpha|$, the analytic continuation of solutions to (7.3) in $\alpha$ shows that the extended point spectrum [10] is given by the extended real point spectrum of the Burgers shock, multiplied by $1 / \alpha^{2}$, thus contained in the stable half plane: the instability therefore is convective in the sense that perturbations to the linearized problem decay pointwise while growing in norm.

Remark 7.1 While the localized waves for $\alpha=0$ discussed in the previous sections correspond to holes in the reaction-diffusion system, the shocks in this section typically correspond to interior or exterior corners. For small $\alpha$, the existence of these corners in the reaction-diffusion system is insured by the results in [3, Section 5]. We may compute the asymptotic slopes

$$
\eta_{ \pm}=\mp\left(\operatorname{Re} \alpha+\frac{\alpha_{2}}{\sqrt{2 \alpha_{1}-\alpha_{2}^{2}}} \operatorname{Im} \alpha+\mathrm{O}\left(|\alpha|^{2}\right)\right),
$$

when $\operatorname{Re} \alpha>0$, in which $\alpha_{j}$ are the coefficients in the quadratic form (2.3). The right-hand side defines a curve

$$
\operatorname{Re} \alpha+\frac{\alpha_{2}}{\sqrt{2 \alpha_{1}-\alpha_{2}^{2}}} \operatorname{Im} \alpha+\mathrm{O}\left(|\alpha|^{2}\right)=0
$$

in the half-plane $\operatorname{Re} \alpha>0$, along which the angles at both $\pm \infty$ change sign, and interior corners change into exterior corners. Interior corners exist in the open region bounded by the curve defined through (7.4) and the imaginary axis, that contains the positive real axis, and exterior corners exist in the interior of its complement. On the boundary between the two regions, the front solutions yield interfaces with zero asymptotic slopes. These are reflection symmetric hole solutions, with an exponential decay of the interface angle at infinity. In particular, the average position of the interface converges at $\pm \infty$ for these holes, whereas it diverges for the holes that we analyzed in the preceding sections.

\section{An example}

Consider the coupled Nagumo-Ginzburg-Landau system

$$
\begin{aligned}
& u_{t}=\Delta u+\left(u-s|a|^{2}\right)\left(1-u^{2}\right), \\
& a_{t}=\Delta a+\left(\kappa+\mathrm{i} \omega_{*}\right) a-u^{2} a-(1+\mathrm{i} \gamma) a|a|^{2},
\end{aligned}
$$

in two space dimensions $x=\left(x_{1}, x_{2}\right)$. Here $u$ and $a$ are real- and complex-valued functions, respectively, and $\kappa, \omega_{*}, \gamma$, and the coupling coefficient $s$ are real parameters. This system 
possesses a steady planar front

$$
(u, a)\left(x_{1}\right)=\left(u_{*}\left(x_{1}\right), 0\right), \quad u_{*}\left(x_{1}\right)=\tanh \left(\frac{x_{1}}{\sqrt{2}}\right) \rightarrow \pm 1, x_{1} \rightarrow \pm \infty .
$$

The one-dimensional linearization about this front is a diagonal operator $\mathcal{L}=\left(L_{u}, L_{a}\right)$ with

$$
L_{u}=\partial_{x_{1} x_{1}}+1-3 u_{*}^{2}, \quad L_{a}=\partial_{x_{1} x_{1}}-u_{*}^{2}+\left(\kappa+\mathrm{i} \omega_{*}\right),
$$

and spectra

$$
\operatorname{spec}\left(L_{u}\right)=(-\infty,-2] \cup\left\{-\frac{3}{2}, 0\right\}, \quad \operatorname{spec}\left(L_{a}\right)=(-\infty,-1] \cup\left\{-\frac{1}{2}\right\}+\left(\kappa+\mathrm{i} \omega_{*}\right) .
$$

At $\kappa=\frac{1}{2}$, the front undergoes a Hopf bifurcation, with a simple pair of eigenvalues $\pm \mathrm{i} \omega_{*}$, and an additional simple zero eigenvalue, due to translation invariance. We denote by $e_{*}$ the real-valued eigenfunction, normalized to have $L^{2}$-norm equal to 1 , of $L_{a}$ associated to the eigenvalue $\mathrm{i} \omega_{*}$, and set $\kappa=\frac{1}{2}+\mu$. The bifurcating solution for $\mu>0$ is a modulated planar traveling front

$$
\begin{aligned}
& u_{\mathrm{mtw}}\left(x_{1}-c t, \omega t\right)=u_{*}\left(x_{1}-c t\right)+\mathrm{O}(\mu), \\
& a_{\mathrm{mtw}}\left(x_{1}-c t, \omega t\right)=r \mathrm{e}^{\mathrm{i} \omega t} e_{*}\left(x_{1}-c t\right)+\mathrm{O}\left(\mu^{3 / 2}\right),
\end{aligned}
$$

in which $c, \omega$, and $r$ depend upon $\mu$,

$$
r^{2}=\frac{1}{e_{3}} \mu+\mathrm{O}\left(\mu^{2}\right), \quad \omega=\omega_{*}-\gamma \mu+\mathrm{O}\left(\mu^{2}\right), \quad c=\frac{\sqrt{2} s}{e_{3}} \mu+\mathrm{O}\left(\mu^{2}\right)
$$

with $e_{3}=\left(e_{*}, e_{*}^{3}\right),(\cdot, \cdot)$ representing the scalar product in $L^{2}(\mathbb{R})$. This solution is accompanied by solutions with periodic modulations in the transverse direction,

$$
\begin{aligned}
& u_{\mathrm{mtw}}\left(x_{1}-c_{k} t, \omega_{k} t-k x_{2}\right)=u_{*}\left(x_{1}-c_{k} t\right)+\mathrm{O}(\mu), \\
& a_{\mathrm{mtw}}\left(x_{1}-c_{k} t, \omega_{k} t-k x_{2}\right)=r_{k} \mathrm{e}^{\mathrm{i}\left(\omega_{k} t-k x_{2}\right)} e_{*}\left(x_{1}-c_{k} t\right)+\mathrm{O}\left(\mu^{3 / 2}\right),
\end{aligned}
$$

with $c_{k}, \omega_{k}$, and $r_{k}$ given by,

$$
r_{k}^{2}=r^{2}-\frac{1}{e_{3}} k^{2}+\mathrm{O}\left(k^{4}\right), \quad \omega_{k}=\omega+\gamma k^{2}+\mathrm{O}\left(k^{4}\right), \quad c_{k}=c-\frac{\sqrt{2} s}{e_{3}} k^{2}+\mathrm{O}\left(k^{4}\right) .
$$

With the Ansatz in Section 2 we find the system of viscous conservation laws (2.1) with $d=\mathrm{id}$, since the diffusion matrix is trivial. We now compute the coefficients $\alpha_{1}$ and $\alpha_{2}$ with the help of the modulated fronts (8.4). These fronts are equilibria of the system of conservation laws with

$$
\eta=0, \quad \rho=-k,
$$

and integration constant

$$
C=\left(-\left(c_{k}-c\right), \omega_{k}-\omega\right)^{T}
$$

Consequently,

$$
c \alpha_{1} k^{2}=c-c_{k}, \quad c \alpha_{2} k^{2}=\omega_{k}-\omega
$$


and from (8.5), we find

$$
\alpha_{1}=\frac{\sqrt{2} s}{e_{3} c}, \quad \alpha_{2}=\frac{\gamma}{c}
$$

We can now compute

$$
\alpha_{2}^{2}-2 \alpha_{1}=\frac{1}{c^{2}}\left(\gamma^{2}-\frac{2 \sqrt{2}}{e_{3}} s c\right)=\frac{1}{c^{2}}\left(\gamma^{2}-\frac{4}{e_{3}^{2}} s^{2} \mu+\mathrm{O}\left(\mu^{2}\right)\right) .
$$

Then, the system is hyperbolic, $\alpha_{2}^{2}>2 \alpha_{1}$, for sufficiently small $\mu$, and falls in the elliptic regime, $\alpha_{2}^{2}<2 \alpha_{1}$, when $\gamma=\mathrm{o}(\sqrt{\mu})$.

Remark 8.1 This example illustrates in an explicit way the significance of the coefficients $\alpha_{j}$ in the transport equation. The quantity $\rho$ stands for the transverse gradient of the phase of the oscillation, typically denoted by $k$ in this Ginzburg-Landau context. The coefficients $\alpha_{j}$ measure the leading-order influence of this transverse wavenumber on the speed of propagation and the frequency of oscillation. The conservation law becomes elliptic, ill-posed in the inviscid limit, when the off-diagonal term in the flux, given by the influence of the transverse modulation of the oscillation on the speed of propagation, dominates the diagonal term, given by the nonlinear dispersion relation in the frequency.

\section{Discussion}

We showed that inherently unstable elliptic conservation laws may well have islands of stability, provided by the basins of attraction for coherent structures. However, we do not know in how far these results are particular to the "integrability" structure, hidden in the transformation to the linear heat equation. While we have shown that the holes persist for general conservation laws with leading quadratic terms in the flux given by (2.3) and that holes moreover yield timeperiodic solutions to the reaction-diffusion system where the shape of the interface diverges logarithmically at infinity [3], stability is a still more subtle issue. The proof of persistence in [3] relies on a geometric blow-up construction, which could well be suited for the analysis of the linearized problem; see for example [11] for such a construction. The difficulty stems from the fact that eigenvalues might pop out of the origin, an edge point of the essential and absolute spectrum.

\section{References}

[1] R. J. Briggs. Electron-steam interaction with plasmas. MIT Press, Cambridge, 1964.

[2] A. Doelman, B. Sandstede, A. Scheel, and G. Schneider. The dynamics of modulated wave trains. Preprint.

[3] M. Haragus and A. Scheel. Corner defects in almost planar interface propagation. Ann. Inst. H. Poincaré, to appear. 
[4] M. Haragus and A. Scheel. Almost planar waves in anisotropic media. Comm. Partial Differential Equations, to appear.

[5] M. Haragus and A. Scheel. A bifurcation approach to non-planar traveling waves in reaction-diffusion systems. GAMM Mitteilungen, to appear.

[6] H.-O. Kreiss and J. Yström. Parabolic problems which are ill-posed in the zero dissipation limit. Math. Comput. Modelling 35 (2002), 1271-1295.

[7] T.-P. Liu and K. Zumbrun. Nonlinear stability of an undercompressive shock for complex Burgers equation. Comm. Math. Phys. 168 (1995), 163-186.

[8] L. Markus. Quadratic differential equations and non-associative algebras. In Contributions to the theory of nonlinear oscillations, Vol. V, Princeton Univ. Press, Princeton, N.J., 1960, 185-213.

[9] B. J. Matkowsky and D. O. Olagunju. Pulsations in a burner-stabilized premixed plane flame. SIAM J. Appl. Math. 40 (1981), 551-562.

[10] B. Sandstede and A. Scheel. Absolute and convective instabilities of waves on unbounded and large bounded domains. Physica D 145 (2000), 233-277.

[11] B. Sandstede and A. Scheel. Evans function and blow-up methods in critical eigenvalue problems. Dyn. Cont. Discr. Dyn. Sys. 10 (2004), 941-964.

[12] D. G. Schaeffer and M. Shearer. The classification of $2 \times 2$ systems of nonstrictly hyperbolic conservation laws, with application to oil recovery. Comm. Pure Appl. Math. 40 (1987), $141-178$. 